\title{
Respuesta de Fragaria mexicana y comunidades microbianas rizosféricas al aumento de temperatura
}

\author{
Adriana Patricia Gamboa-Mendoza1, Julián Delgadillo-Martínez ${ }^{*}$, Juan José Almaraz-Suárez¹, \\ Alejandrina Robledo-Paz ${ }^{2} \&$ Alejandro Alarcón ${ }^{1}$ \\ 1. Postgrado de Edafología. Colegio de Postgraduados, Campus Montecillo. Carretera México-Texcoco km. 36.5. \\ Montecillo, 56230, Estado de México, México; gamboa.adriana@colpos.mx, juliandm@colpos.mx, \\ jalmaraz@colpos.mx, alexala@colpos.mx \\ 2. Postgrado en Recursos Genéticos y Productividad. Colegio de Postgraduados, Campus Montecillo. Carretera México- \\ Texcoco km. 36.5. Montecillo, 56230, Estado de México, México; arobledo@colpos.mx \\ * Correspondencia
}

Recibido 19-IV-2018. Corregido 13-IX-2018. Aceptado 28-I-2019.

\begin{abstract}
Response of Fragaria Mexicana and rhizophere microbial communitites to temperature increase. The earth could experience a warming of $4{ }^{\circ} \mathrm{C}$ in the next one hundred years. This would put at risk the plants that can't adapt. Fragaria mexicana is an endemic plant of temperate forest of Mexico. The response of this wild strawberry to temperature increasing has not been studied and could play an important role for event of global warming. This study determinate the morphological and physiological responses of $F$. mexicana and changes in the $\mathrm{N}_{2}$-fixing bacteria (BFN) population on its roots, due to two environmental conditions with differences of temperature $5.1^{\circ} \mathrm{C}$ : greenhouse (high temperature, TA) and open land (low temperature, TB); and with four free-living nitrogen-fixing bacteria (BFN) treatments: non-inoculated (T), inoculated with strain eight $(\mathrm{CP} 8)$, strain four (CP4), and strains eight and four mixed $(\mathrm{CP} 8+4)$. BFN were isolated from the rhizosphere of F. mexicana and were genetically transformed with reporters to quantify the population at the end of the experiment. $\mathrm{NO}_{3}, \mathrm{NH}_{4}$ and $\mathrm{P}$ and organic matter (MO) in the substrate were determined at beginning and finish of the experiment. Shoot and root dry weight, photosynthetic rate, flowering and fructification starting, flowers and fruits number, were measured. Shoot dry weight and photosynthetic rate were lower in TB than TA, decreasing $3.1 \mathrm{~g}$ and $0.94 \mu \mathrm{mol} \mathrm{m} \mathrm{m}^{-2} \mathrm{~s}^{-1}$, respectively. Root dry weight was $3.0 \mathrm{~g}$ less in TA compared with TB. Number flowers decrease in $40.89 \%$ and number fruits in $38.11 \%$ on TA than TB. F. mexicana plants start flowering 14 days previously in TB than TA. MO in the substrate decrease in TA, while the concentration of $\mathrm{NO}_{3}, \mathrm{NH}_{4}$ and $\mathrm{P}$, increased. Population of BFN was lower in TA. Results obtained indicated that higher temperature promotes the growth of F. mexicana and reduce its reproduction and BNF population associated with its roots.
\end{abstract}

Key words: warming; photosynthesis; plant growth; organic matter; $\mathrm{N}_{2}$-fixing bacteria.

Gamboa-Mendoza, A. P., Delgadillo-Martínez, J., Almaraz-Suárez, J. J., Robledo-Paz, A. \& Alarcón, A. (2019). Respuesta de Fragaria mexicana y comunidades microbianas rizosféricas al aumento de temperatura. Revista de Biología Tropical, 67(1), 94-106.

En México, los bosques templados (oyamel, encino, pino-encino y pino) se caracterizan por una estructura vegetal arbórea caducifolia o perennifolia. En ellos se encuentra gran diversidad vegetal; por ejemplo, contienen $85 \%$ de pinos y $70 \%$ de encinos endémicos (Mittermeier \& Goettsh, 1992; Villaseñor \&
Ortiz, 2014). El estrato herbáceo de los bosques templados representa alrededor del $76 \%$ de las especies, siendo la familia Rosaceae la más numerosa (Sánchez-González, López-Mata, \& Vibrans, 2006). F. mexicana Schltdl. (fresa silvestre) pertenece a esta familia y es endémica de bosques templados de México (Rzedowski 
\& Calderón, 2005; Villaseñor \& Ortiz, 2014; Villaseñor, 2016). F. mexicana, como toda la vegetación secundaria de los bosques templados, se enfrenta a condiciones adversas permanentes debido a fenómenos naturales y antropogénicos. Además, las plantas tienen que adaptarse a los cambios ambientales de los últimos años, asociados al calentamiento global (Torres, 2004; Pawar \& Rothkar, 2015).

El calentamiento global puede causar efectos diferenciales sobre la productividad, fotosíntesis, respiración, crecimiento y ciclo de vida de las plantas; emergencia de la semilla, número de brotes, área foliar, número de hojas, de flores o inflorescencias, entre otros; dependiendo de la especie, el genotipo y la región. Algunas plantas podrían aumentar su productividad por un aumento de temperatura, mientras que en otras se podría superar el límite térmico de crecimiento (Struik, 2007; Huang, von Lampe, \& Tongeren, 2011; Zhang, Cai, Beach, \& McCarl, 2014; Lizana, Avila, Tolaba, \& Martínez, 2017).

Por ejemplo, incrementos en la temperatura aumentan la fotosíntesis en Vitis vinifera (Greer \& Weedon, 2012); los rendimientos en Solanum tuberosum L. (Lizana et al., 2017) y Zea mays L. (Li, Takahashi, Suzuki, \& Kaiser, 2014). En otros casos se reporta disminución en el rendimiento de Oryza sativa L. (Sheehy, Mitchell, \& Ferrer, 2006), Triticum aestivum L. (Asseng, Foster, \& Turner, 2011), Zea mays L. (Sierra, Brisson, Ripoche, \& Déque, 2010; Li et al., 2014), Phaseolus vulgaris L. (Porch \& Jahn, 2001) y Olea europaea L. (BenllochGonzález et al., 2016). Además, se ha observado una disminución en la tasa de asimilación de $\mathrm{CO}_{2}(A)$, la tasa de conductancia estomática $\left(\mathrm{g}_{\mathrm{s}}\right)$, la viabilidad, arquitectura y germinación del polen; y una mayor retención de polen en las anteras (Porch \& Jahn, 2001; Young, Wilen \& Bonham-Smith, 2004; Greer \& Weedon, 2012; Hartsan, Pavlovic, Chiu, Sultmanis, \& Sage, 2013; Prasad \& Djanaguiraman, 2014; Sage et al., 2015).

Poco se conoce de los efectos del cambio climático en la vegetación secundaria de los bosques (Bonilla-Valencia et al., 2017) y no se tienen reportes de $F$. mexicana en bosques de oyamel. El género Fragaria a nivel mundial tiene importancia económica y alimenticia, debido a que muchas especies tanto comerciales como silvestres, producen frutos comestibles y son utilizadas por las comunidades locales en diversas regiones (Rzedowski \& Calderón, 2005; Barrie, 2015). En especies cultivadas de Fragaria hay resultados controversiales en el crecimiento y producción de frutos, debido al calentamiento global. Sønsteby, Solhaug \& Heide (2016), encontraron que un aumento de temperatura de $12{ }^{\circ} \mathrm{C}$ incrementa la materia seca de las hojas, tasa relativa de crecimiento, tasa de asimilación neta, área foliar, los días para la antesis y el número de flores de Fragaria $x$ ananassa variedad Sonata. La variedad Toyonoka presentó una disminución en el número de frutos, peso fresco y tamaño de los frutos al aumentar la temperatura (Ledesma \& Kawabata, 2016).

Asimismo, el impacto del cambio climático en los microorganismos del suelo es importante ya que estos intervienen en los ciclos biogeoquímicos (como las bacterias fijadoras de $\mathrm{N}$ atmosférico); movilización, descomposición y flujo de nutrimentos; y en la interacción con los ecosistemas terrestres a través de las plantas, entre otros, promoviendo el crecimiento vegetal (Urbanová, Snajdr, \& Baldrian, 2015). Egamberdiyeva \& Höflich (2003), observaron que Mycobacterium sp., una rizobacteria que promueve el crecimiento en trigo, disminuye el tamaño de su población en las raíces, al aumentar la temperatura de 16 a $26{ }^{\circ} \mathrm{C}$, mientras que la población de Mycobacterium bullata aumenta. Según Compant, van der Heijden, \& Sessitsch (2010), el efecto del incremento de temperatura sobre las bacterias promotoras de crecimiento depende del genotipo y de las condiciones ambientales del lugar de aislamiento, por lo que bacterias aisladas de lugares con temperatura altas pueden responder mejor a los aumentos de temperatura. Sin embargo, no existe una respuesta concluyente en el comportamiento de los microorganismos. Diferentes autores han encontrado que la biomasa microbiana y la actividad enzimática de los suelos 
puede disminuir (Tripathi, Abhilash, Singh, Singh, \& Patra, 2015) o aumentar (Sofi et al., 2016), con el incremento de la temperatura.

En este contexto, esta investigación tuvo como objetivo identificar los efectos de la temperatura y la radiación solar en plantas de fresa silvestre y sobre las comunidades de bacterias fijadoras de nitrógeno de vida libre asociadas; así como los cambios en la materia orgánica y los nutrientes del sustrato. Un acercamiento a los cambios en la relación planta-microorganismos-suelo por efecto del aumento de temperatura, puede brindar información clave ante escenarios de cambio climático.

\section{MATERIALES Y MÉTODOS}

Se recolectaron 56 plantas de fresa silvestre (Fragaria mexicana) en una zona de bosque de oyamel en el Monte Tláloc, Texcoco, Estado de México (19³0'14" N \& 98 53'2" W); a $3121 \mathrm{~m}$ de altitud, con una temperatura anual entre 10 a $14{ }^{\circ} \mathrm{C}$ y una precipitación anual entre 900 a $1200 \mathrm{~mm}$ (Ortiz-Solorio \& Cuanalo de la Cerda, 1977). Cada planta se dejó con aproximadamente $500 \mathrm{~g}$ de suelo rizosférico. Posteriormente, colocadas en un invernadero en Texcoco, Estado de México; y se trasplantaron a bolsas de polietileno conteniendo $2 \mathrm{~kg}$ de sustrato (50\% de turba y $50 \%$ de arena de río lavada). Todas las plantas se mantuvieron con las mismas condiciones (temperatura mínima de $14{ }^{\circ} \mathrm{C}$ y máxima de $23^{\circ} \mathrm{C}$ y radiación promedio de $350 \mu$ moles $\mathrm{m}^{-2} \mathrm{seg}^{-1}$ ) durante 18 meses, para confirmar su capacidad de crecer en condiciones de invernadero a $2220 \mathrm{~m}$ de altitud.

Se estableció un experimento factorial que incluía dos niveles de temperatura y cuatro niveles para bacterias fijadoras de $\mathrm{N}$ atmosférico (BFN), produciendo ocho tratamientos con siete réplicas cada uno, distribuidos en un diseño completamente al azar. Las dos condiciones de temperatura correspondieron a invernadero (temperatura alta, TA) y campo abierto (temperatura baja, TB). En invernadero la temperatura promedio fue de $19.6^{\circ} \mathrm{C}$, y la radiación fotosintéticamente activa de $341.3 \mu$ moles $\mathrm{m}^{-2}$ $\mathrm{seg}^{-1}$, con un rango de temperatura de $9.5{ }^{\circ} \mathrm{C}$ (día-noche), y de radiación de $511.39 \mu$ moles $\mathrm{m}^{-2} \mathrm{seg}^{-1}$, entre las 10:30 y 13:30. En campo abierto la temperatura media fue de $14.5{ }^{\circ} \mathrm{C}$ y la radiación de $630.4 \mu$ moles $\mathrm{m}^{-2} \mathrm{seg}^{-1}$, con un rango de temperatura día-noche de $20.4^{\circ} \mathrm{C}$ y de radiación de $579.19 \mu$ moles $\mathrm{m}^{-2} \mathrm{seg}^{-1}$, entre las 10:30 y 13:30. La temperatura se registró cada hora con datalogger durante todo el experimento (Watchdog 1450, Marca Spectrum Technologies Inc.). La radiación solar se midió en cuatro fechas distintas, durante tres momentos del día con un fotosensor de varilla (Marca Licor, LI-191R). El segundo factor se relacionó con la inoculación de BFN aisladas de la rizósfera de fresa silvestre y tuvo cuatro niveles: plantas sin inocular (T), plantas inoculadas con la cepa cuatro (CP4), con la cepa ocho (CP8) y con ambas $(\mathrm{CP} 4+8)$.

Propiedades químicas del sustrato: $\mathrm{Al}$ inicio y final del experimento, se tomó una muestra compuesta del sustrato (suelo rizosférico, turba y arena), se secó a temperatura ambiente por $15 \mathrm{~d}$, se tamizó en malla 60 y se analizaron sus propiedades químicas: $\mathrm{pH}$ (1:2 en agua), conductividad eléctrica, materia orgánica (MO) (Walkley \& Black, 1934), P (Bray \& Kurtz, 1945), $\mathrm{N}^{-\mathrm{NO}_{3}}$ y N-NH 4 determinados por destilación por arrastre de vapor (Baker, 1969). Se calcularon las diferencias entre las propiedades químicas iniciales y finales en cada uno de los tratamientos.

Aislamiento de bacterias fijadoras de $\mathbf{N}$ atmosférico (BFN): Las BFN se aislaron de la endorizósfera de las plantas de fresa silvestre y se sembraron en tubos con medio de cultivo semisólido sin fuente de N (Rennie, 1981). Los tubos se incubaron a $28^{\circ} \mathrm{C}$ por $10 \mathrm{~d}$. Las bacterias que crecieron en la zona microaerofílica (media luna por debajo de la superficie del medio de cultivo) se re-sembraron en medio sólido sin fuente de $\mathrm{N}$ en cajas de Petri. Un total de 11 cepas fueron aisladas. Finalmente, las cepas cuatro y ocho fueron seleccionadas por su crecimiento rápido en el medio de cultivo a las $24 \mathrm{~h}$. 
Transformación genética de bacterias e inoculación: Con la finalidad de poder rastrear a las bacterias inoculadas mediante un gen reportero y por su resistencia a antibióticos; las cepas fueron transformadas genéticamente con vectores distintos. La cepa cuatro se transformó con el vector pMP7604 que llevaba el gen de resistencia a tetraciclina (Tet) y el gen reportero DsRed (proteína rojo fluorescente), mientras que la cepa ocho se transformó con el vector p519gfp que contenía el gen de resistencia a kanamicina (KanR) y el gen reportero gfp (proteína verde fluorescente) (Sambrook, Maniatis, \& Fritsch, 1989; Lodish et al., 2000).

Para elaborar el inoculante, cada cepa transformada fue cultivada en caldo nutritivo con los antibióticos respectivos e incubada por 24 h en agitación. El inóculo se centrifugó a 7 $000 \mathrm{rpm}$ por $15 \mathrm{~min}$. En cámara de flujo laminar se descartó el sobrenadante y se agregaron $25 \mathrm{~mL}$ de agua destilada estéril. Finalmente, al momento del trasplante se aplicaron $5 \mathrm{~mL}$ de inóculo por planta con una concentración celular aproximada de $3 \times 10^{9}$ células $\mathrm{mL}^{-1}$.

Colonización de las raíces de fresa silvestre por BFN: La población de BFN inoculadas que permanecieron en la rizósfera de fresa silvestre al final del experimento se cuantificó por el método de diluciones y cuenta viable en caja de Petri. De cada planta se separó $1 \mathrm{~g}$ de raíz con suelo rizosférico para realizar las diluciones decimales. Se sembraron $100 \mu \mathrm{L}$ de las diluciones $10^{-3}$ y $10^{-4}$ en cajas de Petri con agar nutritivo más antibiótico, tetraciclina para cepa cuatro y kanamicina para cepa ocho.

Parámetros fotosintéticos: la tasa de asimilación de $\mathrm{CO}_{2}(A)$ se evaluó a los 62, 113 y 130 d después de la inoculación (ddi) con el equipo portátil CIRAS-2 (Marca PP Systems, Modelo PCL-6 Universal Leaf Cuvette, Amesbury, Massachusetts, Estados Unidos de América), tomando el foliolo central de la hoja compuesta, completamente expandido y sin daños por plagas o enfermedades. Las condiciones de la cámara fueron temperatura de $22{ }^{\circ} \mathrm{C}$, humedad relativa de $18 \%, 450 \mathrm{ppm}$ de $\mathrm{CO}_{2}$ y una densidad de flujo de fotones de $1 \mathrm{mmol} \mathrm{m}^{-2} \mathrm{~s}^{-1}$.

Variables del crecimiento y reproductivas: Se llevó a cabo la cosecha de las plantas a los 213 ddi; se separó la raíz de la parte aérea y se contó el número de flores y frutos. $\mathrm{El}$ material vegetal se secó en un horno a $70{ }^{\circ} \mathrm{C}$ durante $48 \mathrm{~h}$ y posteriormente se pesó en una balanza analítica.

Análisis estadístico: Para el análisis de las variables del suelo (contenido de materia orgánica, nitratos, amonio y $\mathrm{P}$ extractable) se realizaron histogramas de diferencias contenido inicial-final. Después de determinar que los resultados no se ajustan a una distribución normal (asimetría), se realizó una prueba de Mann-Whitney-Wilcoxon pareada por tratarse de una muestra con tres repeticiones y sin observaciones anómalas, utilizando el paquete estadístico R vers. 3.5.1 (R Development Core Team, 2018). Las variables fisiológicas, reproductivas y de colonización de las raíces de fresa silvestre por BFN fueron sometidas a ANOVA de dos vías para los siguientes factores: temperatura y BFN, con comparaciones entre medias a posteriori con la prueba de Tukey's HSD ( $\alpha=$ 0.05 ), utilizando el paquete SAS (SAS Institute Inc., 2002).

\section{RESULTADOS}

Análisis de las propiedades químicas del sustrato: Las propiedades químicas iniciales fueron $5.89 \mathrm{pH}, 0.77 \mathrm{dSm}^{-1}$ conductividad eléctrica, $8.0 \%$ de materia orgánica, $3 \mathrm{mg}$ de $\mathrm{P}$ $\mathrm{kg}^{-1}, 29 \mathrm{mg}$ de $\mathrm{NO}_{3} \mathrm{~kg}^{-1}$ y $15 \mathrm{mg}$ de $\mathrm{NH}_{4} \mathrm{~kg}^{-1}$ de suelo. En ambas condiciones de temperatura hubo disminución $(\mathrm{P}<0.01)$ en el contenido de $\mathrm{MO}$ al final del experimento, pero en la TA la reducción fue mayor (Cuadro 1). Esta reducción en el contenido de $\mathrm{MO}$ indica un proceso de mineralización de esta, el cual es mayor cuando la temperatura es más alta, lo que concuerda con la mayor disponibilidad de $\mathrm{N}$ en forma de $\mathrm{NO}_{3}$ y de $\mathrm{NH}_{4}$; así como para el $\mathrm{P}$ disponible (Cuadro 1). 
CUADRO 1

Diferencia en las propiedades químicas del sustrato al iniciar y al finalizar el experimento en $F$. mexicana inoculada con bacterias fijadoras de $\mathrm{N}$ atmosférico (BFN) y en dos condiciones

TABLE 1

Differences in the chemical properties of the substrate at the end of the experiment in F. mexicana inoculated with free-living nitrogen-fixing bacteria (BFN) and grown under two environments

\begin{tabular}{|c|c|c|c|c|c|}
\hline \multirow{2}{*}{ Condición } & \multirow{2}{*}{ BFN } & \multirow{2}{*}{$\mathrm{MO}^{*}(\%)$} & $\mathrm{N}-\mathrm{NO}_{3}{ }^{*}$ & $\mathrm{~N}-\mathrm{NH}_{4}{ }^{*}$ & $\mathrm{P}^{*}$ \\
\hline & & & \multicolumn{3}{|c|}{ (mg kg-1 de suelo) } \\
\hline \multirow[t]{4}{*}{ TB } & Sin inocular & -3.8 & 11 & 0 & 38 \\
\hline & $\mathrm{CP} 4$ & -3.7 & 10 & 1 & 12 \\
\hline & CP8 & -3.5 & 4 & 1 & 65 \\
\hline & $\mathrm{CP} 4+8$ & -4.2 & 6 & 1 & 30 \\
\hline \multirow[t]{6}{*}{ TA } & Sin inocular & -5.0 & 14 & 3 & 32 \\
\hline & $\mathrm{CP} 4$ & -5.6 & 14 & 1 & 24 \\
\hline & $\mathrm{CP} 8$ & -3.8 & 18 & 3 & 39 \\
\hline & $\mathrm{CP} 4+8$ & -3.2 & 15 & 1 & 41 \\
\hline & Valor de U & 300 & 0 & 21 & 243 \\
\hline & $\mathrm{P}$ & $1.8 \times 10^{-5}$ & $1.8 \times 10^{-5}$ & $2.0 \times 10^{-4}$ & $7.8 \times 10^{-3}$ \\
\hline
\end{tabular}

Números positivos indican aumento y negativos indican reducción de la concentración del sustrato con respecto a la concentración inicial. * = Las diferencias entre el contenido inicial menos el contenido final son estadísticamente diferentes (Mann-Whitney-Wilcoxon, $\alpha=0.01$ ). TB= temperatura baja, $\mathrm{TA}=$ temperatura alta.

Positive and negative numbers indicate an increase and decrease concentration of analytes respectively, with the initial concentration as reference.

* Represents statistically significant differences between initial and final contents of chemical properties (Mann-WhitneyWilcoxon, $\alpha=0.01$ ). $\mathrm{TB}=$ low temperature, $\mathrm{TA}=$ high temperature.

\section{Colonización de las raíces de fresa silves-} tre por BFN: Independientemente de la cepa inoculada, en las condiciones TA la población microbiana de $\mathrm{BFN}$ fue menor $(\mathrm{F}=5.19$, g.l. $=$ $1, \mathrm{P}<0.05)$ que en la TB a los 213 ddi $\left(3.5 \times 10^{4}\right.$ y $8.0 \times 10^{4} \mathrm{UFC} \mathrm{g}^{-1}$ de sustrato respectivamente). Ambas cepas, aisladas de las raíces de fresas silvestres e inoculadas en este experimento, disminuyeron el tamaño de su población $(\mathrm{F}=$ $5.19, \mathrm{gl}=1, \mathrm{P}<0.05)$ con el aumento de temperatura. La CP8 mostró una disminución de $27.99 \%$ y la CP4 de $21.82 \%$. Cuando se hizo la inoculación conjunta de las cepas, la reducción por efecto de la TA fue aún mayor $(70.10$ y $76.73 \%$, para CP8 y CP4, respectivamente). La cepa que se estableció mejor en TA y TB, fue la CP8 (hasta $2.2 \times 10^{5} \mathrm{UFC} \mathrm{g}^{-1}$ de sustrato, Fig. 1), aunque sin diferencias significativas. Las dos cepas al inocularse juntas $(\mathrm{CP} 8+4)$ y solas, mostraron una mayor población en TB; mientras que, en TA, la población de las cepas en el tratamiento $\mathrm{CP} 8+4$ es aún menor que cuando estás se encuentran solas (Fig. 1), sin presentarse diferencias significativas.

Tasa de asimilación de $\mathrm{CO}_{2}(A): A$ fue afectada por los dos factores, temperatura, BFN y por la interacción entre ambos factores $(\mathrm{F}=2.93$, g.l. $=3, \mathrm{P}<0.05)$. Las plantas del testigo absoluto presentaron el valor más bajo de $A$ para TA y TB, mientras que las plantas inoculadas con $\mathrm{CP} 4+8$, presentaron el valor más alto (Fig. 2).

Número de flores y frutos: La floración a TB inició 120 ddi y TA a los 134 d; en tanto que el número de flores siempre fue mayor $(\mathrm{F}=$ 93.86, g.1. $=1, \mathrm{P}<0.05$ ) en TB que en TA (Fig. 3A y Fig. 3B). El factor BFN y la interacción de la condición con las BFN no presentaron efecto sobre la floración y fructificación $(\mathrm{F}=0.9$, g.l. $=3, \mathrm{P}>0.05$ y $\mathrm{F}=0.09$, g.l. $=3, \mathrm{P}>0.05$ ) pero los valores en todos los muestreos fueron menores en el testigo sin inocular (Fig. 3A, 


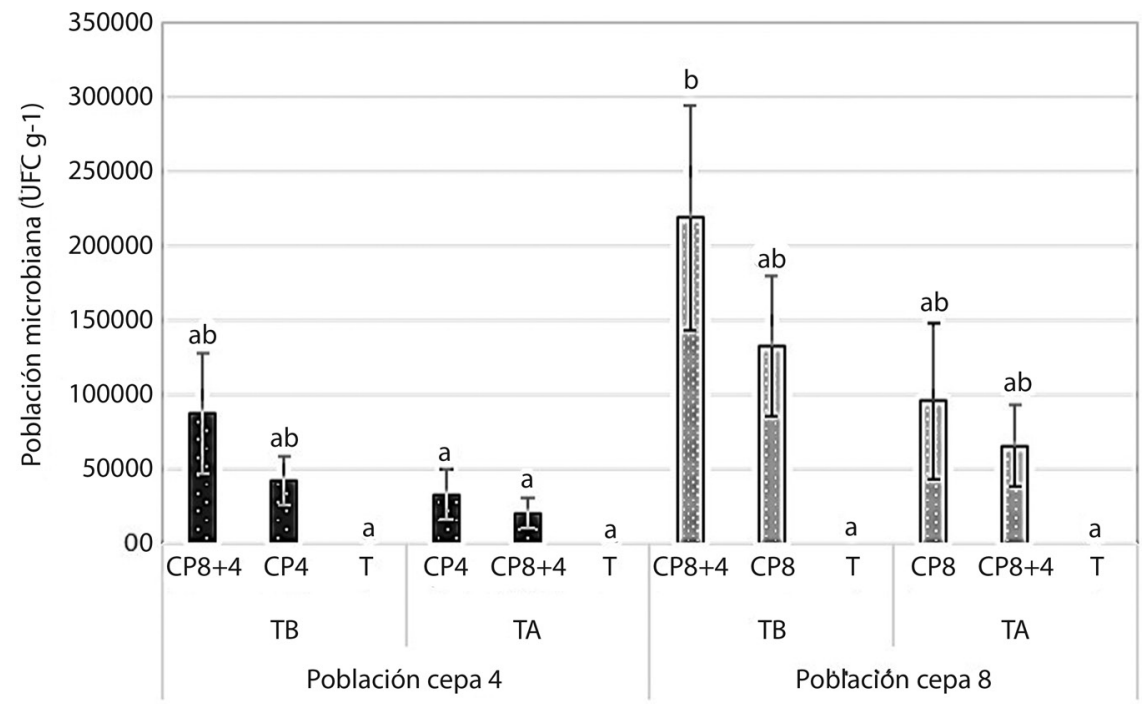

Fig. 1. Población de bacterias fijadoras de nitrógeno (BFN) recuperadas a los 213 ddi en dos condiciones: alta (TA) y baja (TB) temperatura, en los tratamientos inoculados con $\mathrm{CP} 8, \mathrm{CP} 4$ y CP8+4. Letras diferentes indican diferencias significativas entre tratamientos (Tukey, $\mathrm{P}=0.05$ ).

Fig. 1. Free-living nitrogen-fixing bacteria (BFN) population recovered 213 days after beginning the experiment under high (TA) and low (TB) temperature, for the treatments inoculated with CP8, CP4 and CP8+4. Letters indicate statistical differences (Tukey, $\mathrm{P}=0.05$ ).

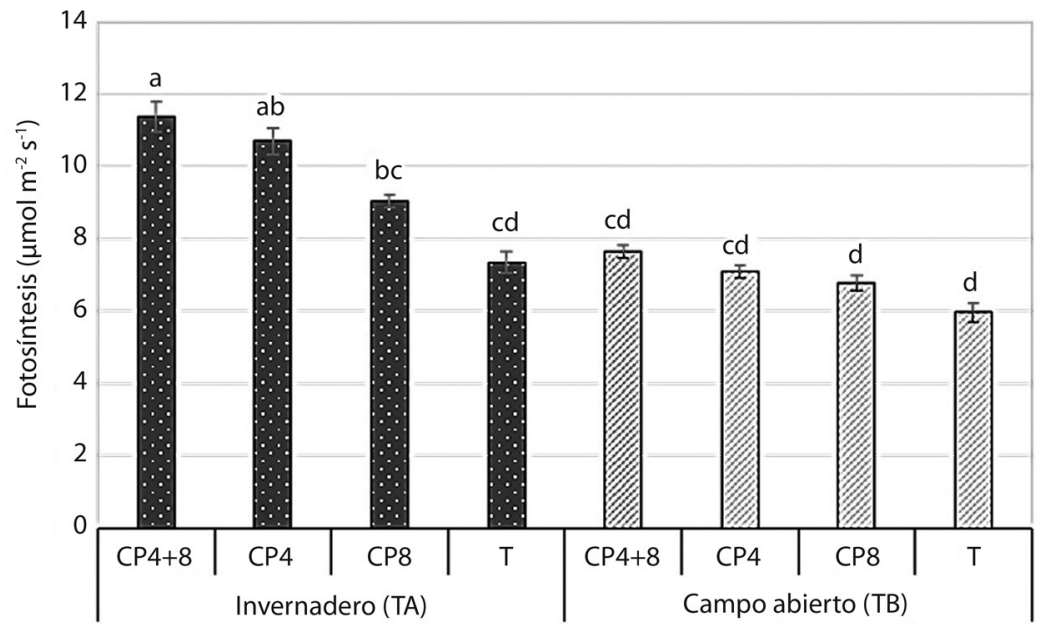

Fig. 2. Tasa de asimilación de $\mathrm{CO}_{2}(A)$ en F. mexicana sin inocular (T) e inoculada con la cepa ocho (CP8), cuatro (CP4) y ambas $(\mathrm{CP} 8+4)$, en dos condiciones: alta (TA) y baja (TB) temperatura. Letras diferentes indican diferencias significativas entre tratamientos (Tukey, $\mathrm{P}=0.05$ ).

Fig. 2. Assimilation rate of $\mathrm{CO}_{2}(A)$ in non-inoculated (T) and inoculated treatments $F$. mexicana with strain eigth (CP8), four (CP4), and mixed (CP8 + 4) under high (TA) and low (TB) temperature. Letters indicate statistical differences (Tukey, $\mathrm{P}=0.05)$. 

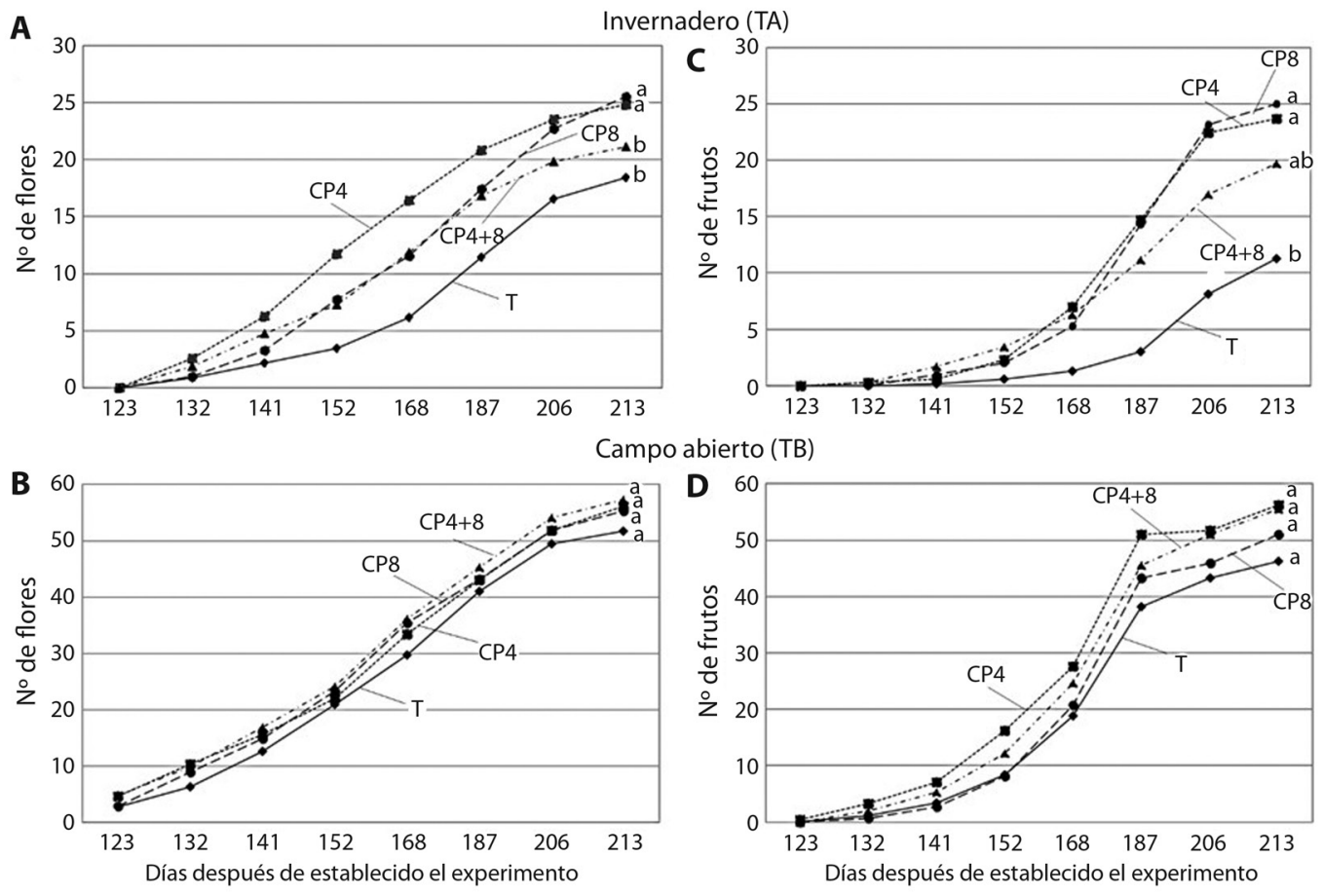

Fig. 3. Número de flores (A, B) y frutos (C, D) en fresa silvestre sin inocular (T) e inoculadas con la cepa ocho (CP8), cuatro (CP4) y ambas (CP8+4) en dos condiciones: alta (TA) y baja (TB) temperatura. Letras diferentes indican diferencias significativas entre tratamientos (Tukey, $\mathrm{P}=0.05$ ).

Fig. 3. Number of flowers and fruits in non-inoculated $(\mathrm{T})$ and inoculated treatments wild strawberries with strain eigth (CP8), four (CP4), and mixed (CP8 + 4) under high (TA) and low (TB) temperature. Letters indicate statistical differences (Tukey, $\mathrm{P}=0.05$ ).

Fig. 3B, Fig. 3C y Fig. 3D). Los frutos se desarrollaron desde 132 ddi en TB y para TA $14 \mathrm{~d}$ después de TB. El número de frutos siempre fue mayor $(\mathrm{F}=83.98$, g.l. $=1, \mathrm{P}<0.05)$ en TB que en TA (Fig. 3C y Fig. 3D) y las diferencias fueron más marcadas a partir de 168 ddi.

Peso seco de la parte aérea y de la raíz: Las plantas en condiciones de TA presentaron una mayor acumulación de biomasa seca en la parte aérea $(\mathrm{F}=18.10$, g.l. $=1, \mathrm{P}<0.05)$ con respecto a las plantas de TB. Aunque no se observó un efecto $(\mathrm{F}=0.76$, g.l. $=3, \mathrm{P}<$ $0.05)$ de las BFN y de la interacción de los dos factores. Los testigos sin inocular presentaron el menor peso seco de la parte aérea en ambas condiciones de temperatura (Fig. 4). De manera contraria, la acumulación de materia seca en la raíz fue menor $(\mathrm{F}=20.90$, g.l. $=1, \mathrm{P}<0.05)$ en TA que en TB (Fig. 4).

\section{DISCUSIÓN}

Al final del experimento el contenido de $\mathrm{MO}$ en el sustrato se redujo, lo que indica un proceso de mineralización que se acrecentó en TA. Así mismo, se determinó que la disponibilidad de $\mathrm{NO}_{3}, \mathrm{NH}_{4}$ y $\mathrm{P}$ fue mayor en TA. La velocidad de descomposición de la MO es sensible a los cambios de temperatura del suelo, principalmente la fracción lábil, la cual está influenciada por la diversidad de plantas que se encuentran en el ecosistema que afectan directamente la calidad del sustrato (presencia de ceras, lignina y resinas recalcitrantes) y la 


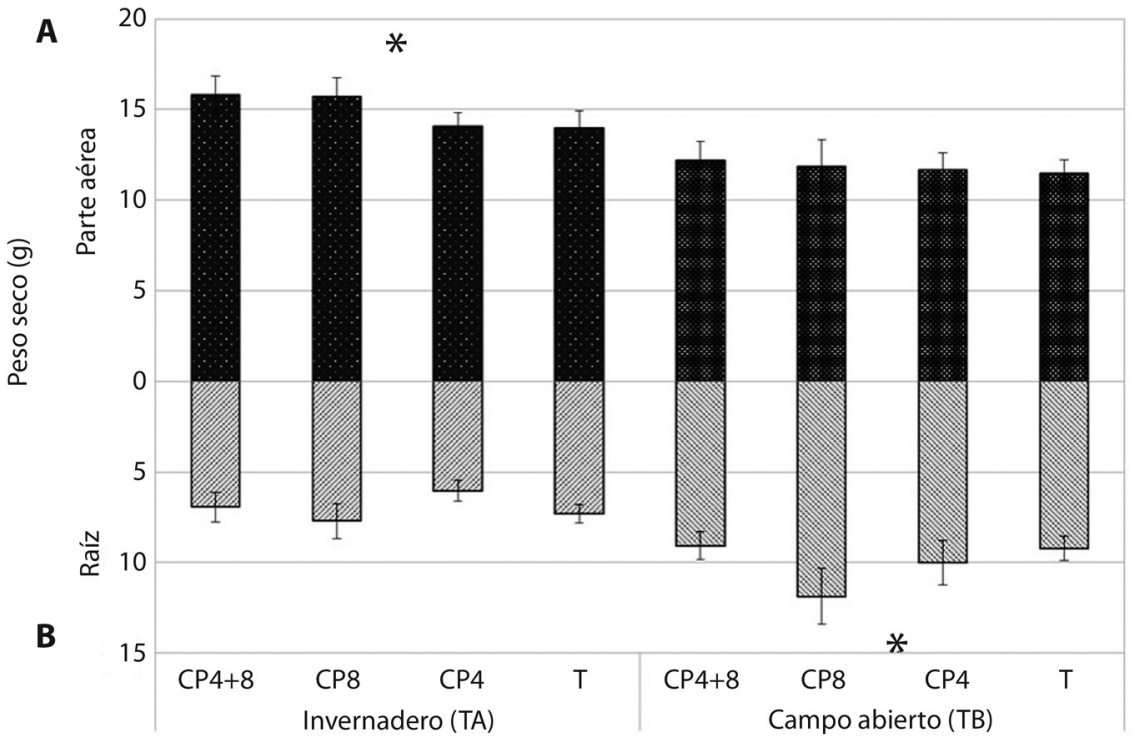

Fig. 4. Peso seco de la parte aérea (A) y de la raíz (B) de fresa silvestre sin inocular (T) e inoculada con las cepas ocho (CP8), cuatro $(\mathrm{CP} 4)$ y ambas $(\mathrm{CP} 8+4)$, en dos condiciones: alta (TA) y baja (TB) temperatura. * indica diferencias estadísticas significativas entre TA versus $\mathrm{TB}$ (Tukey $\mathrm{P}=0.05$ ).

Fig. 4. Shoot and root dry weights of wild strawberry in non-inoculated $(\mathrm{T})$ and inoculated treatments with strain eigth (CP8), four (CP4), and mixed (CP8 + 4) under high (TA) and low (TB) temperature. * indicate statistical differences on TA versus $\mathrm{TB}$ (Tukey $\mathrm{P}=0.05$ ).

composición de las comunidades de microorganismos del suelo (Wetterstedt, Persson \& Agren, 2010; Wang, Zhou, Xu, Ruan \& Wang, 2013; Li et al., 2017). Li, Cheng, Luo, Xu, \& $\mathrm{Xu}$ (2017), reportan un incremento en la tasa de mineralización de la fracción lábil de $\mathrm{MO}$ de 2 a $31 \%$, con un incremento de temperatura de 5 a $25^{\circ} \mathrm{C}$ en bosque de coníferas.

La mayor disponibilidad de $\mathrm{N}$ en el sustrato debido a la mineralización de la MO inhibió la población de BFN; ya que a los 213 ddi, CP4 y CP8 disminuyeron, especialmente en TA. De acuerdo con Shen, Xu, Chi, Yu, \& Wan (2014) el calentamiento global ocasiona cambios drásticos en las comunidades microbianas $\mathrm{y}$ en el caso de BFN, la disponibilidad de $\mathrm{N}$ en el suelo reduce la población de estos; ya que la fijación de $\mathrm{N}$ atmosférico es un proceso energéticamente caro e inhibido cuando el $\mathrm{N}$ se puede obtener de otras fuentes (Paul, 2007; Merlo, Reyna, Abril, Amé, \& Genti-Raimondi, 2014). Adicionalmente, el peso seco de la raíz fue menor en TA, lo que indica que la mayor disponibilidad de nutrimentos redujo el crecimiento del sistema radical. La asimetría encontrada en esta investigación, en raíz/parte área, es un efecto determinado por la disponibilidad de nutrientes y agua (Gregory \& Kirkegaard 2017). En el caso de baja disponibilidad de $P$ en el suelo, se promueve el crecimiento de las raíces hacia superficie del suelo, dado que el $\mathrm{P}$ es inmóvil; mientras que el $\mathrm{N}$, al ser nutriente bastante móvil, la raíz crece con abundante proliferación de pelos radicales a lo largo del perfil del suelo, con el fin de evitar la lixiviación de este (Gregory \& Kirkegaard 2017).

De la misma manera, a mayor temperatura se incrementó $A$ y el peso seco de la parte aérea. Lo anterior puede asociarse, en parte, a la mayor disponibilidad de $\mathrm{N}$ y $\mathrm{P}$ en el suelo, asociada a mayor mineralización de la $\mathrm{MO}$ en TA. Asimismo, la ganancia en peso seco está relacionada directamente con la actividad fotosintética. Para Fragaria vesca L., se ha encontrado que un incremento en la temperatura entre 20 y $25^{\circ} \mathrm{C}$, logra un máximo de eficiencia 
fotosintética relacionada con una mayor biomasa seca y un comportamiento estomatal eficiente (Chabot, 1978; Tkemaladze \& Makhashvili, 2016). A temperaturas por debajo de $15{ }^{\circ} \mathrm{C}$, la $A$ disminuye de manera rápida por el cierre de estomas y el crecimiento vegetal se detiene (Chabot, 1978; Robert, Gissen, \& Pétel, 1999; Åström, Metsovuori, Saarinen, Lundell, \& Hännninen, 2015; Saarinen, Rasmus, Lundell, Kauppinen, \& Hänninen, 2016). Sin embargo, Rothstein \& Zack (2001) determinaron que algunas plantas de bosques deciduos tienen la característica de acelerar de manera inmediata su capacidad fotosintética en el momento que la temperatura aumenta.

Prasad, Thomas, \& Narayanan (2017) concluyeron que el calentamiento global, principalmente las altas temperaturas, tiene efectos más evidentes en aspectos reproductivos que vegetativos de las plantas, debido a que altas temperaturas puede afectar la viabilidad y morfología del polen, resultando en una disminución en la producción de semillas. Sønsteby et al. (2016) mencionan que el crecimiento de plantas de fresa en condiciones controladas aumentó de manera directa con la temperatura, mientras que el proceso de floración requiere días cortos y bajas temperaturas. De acuerdo con lo anterior, en el presente trabajo el inicio de la floración ocurrió antes (14 d) en TB que en TA; y el número de flores y frutos fue mayor en TB. Lo anterior se ha asociado a promotores de genes de floración (FaFT3) inducidos por temperatura y fotoperiodo, que se regulan en los meristemos apicales de fresa durante los días cortos y temperaturas bajas (Nakano, Higuchia, Yoshidab, \& Hisamatsua, 2015). Una intrincada red de reguladores (epi) genéticos reaccionan a las condiciones ambientales para desencadenar la floración (Blümel, Dally \& Jung, 2015). Específicamente en el género Fragaria, Yanagi, Takanori, \& Ncbuyuki, (2006) encontraron que la iniciación floral, la emergencia de la inflorescencia y la producción de estolones suceden en tres temporadas durante el año. La iniciación floral se desata con fotoperiodo largo y temperaturas mayores a $20{ }^{\circ} \mathrm{C}$ en $90 \%$ de las plantas, pero, se induce en el $100 \%$ de las plantas con $8 \mathrm{~h}$ de luz diaria y temperatura menor a $20{ }^{\circ} \mathrm{C}$. Lo anterior indica que la fresa es una planta de día largo que se comporta como de día corto facultativa con temperaturas bajas.

La disminución de la fructificación observada en TA también fue reportada por Ledesma \& Kawata (2016) en dos cultivares de Fragaria $x$ ananassa Duch., Nyoho y Toyonoka, en los cuales se observó una respuesta similar al aumento de temperatura, con una reducción en el porcentaje total de frutos para los dos cultivares. Por lo anterior, los autores concluyeron que, en este tipo de fresa cultivada a campo abierto, un evento de temperatura alta disminuye la producción de frutos.

Fragaria mexicana crece en el estrato herbáceo de bosques de oyamel, donde la temperatura oscila entre los 10 a $14{ }^{\circ} \mathrm{C}$, con una radiación solar baja, debido a la vegetación arbórea existente (Ortiz-Solorio \& Cuanalo de la Cerda, 1977; Rzedowski \& Calderón, 2005). En este caso, el aumento en la temperatura en $5.1{ }^{\circ} \mathrm{C}$ provocó un mayor crecimiento en la parte aérea, pero con una disminución en el crecimiento de la raíz y en el número de flores y frutos de la planta. El incremento de temperatura modificó el patrón de la floración y fructificación. Es muy probable que, en eventos de aumento de temperatura en los bosques de oyamel, la fresa silvestre sea impactada de manera significativa, especialmente en su reproducción, lo que podría darles una desventaja con respecto a otras plantas del estrato herbáceo, o iniciar un evento de migración de la especie a zonas con hábitats más fríos. Esto podría evitarse ya que puede reproducirse vegetativamente, lo que puede llegar a permitir una migración de esta planta a sitios de temperatura más alta, aunque una predicción en este sentido es complicada (Liu, Mu, Niklas, Li, \& Sun, 2012).

Al respecto, Tkemaladze \& Makhashvili (2016) mencionan que las plantas de clima frío en Europa han estado migrando a las zonas más altas de 1 a 4 m cada diez años y el calentamiento global puede ocasionar serios problemas en la persistencia de éstas. Aunque Vanha-Majamaa, 
Shorohova, Kushnevskava, \& Jalonen (2017) afirman que las especies más resilientes a los disturbios son las herbáceas, comparadas con otros grupos de plantas de la vegetación secundaria como musgos, líquenes, helechos y hepáticas. Otro escenario posible indicaría la existencia de genotipos de fresa silvestre que se pudiesen adaptar al aumento de temperatura, pues como lo indican Sønsteby, Opstada, \& Heideb (2013), Noruega cuenta con genotipos de fresa (Korona, Polka y Sonata) de clima frío, en los cuales la temperatura baja es un factor limitante para la inducción floral y no el fotoperiodo.

Las BFN se redujeron por el incremento de temperatura y $\mathrm{N}$ disponible (mayor mineralización de la MO). La población microbiana recuperada en TA fue mayor para $\mathrm{CP} 8,53.6 \mathrm{x}$ $10^{3} \mathrm{UFC} \mathrm{g}^{-1}$, que para CP4, $17.7 \times 10^{3} \mathrm{UFC} \mathrm{g}^{-1}$ de sustrato. De las dos cepas aisladas del suelo rizosférico de $F$. mexicana, CP8 podría tener mejor capacidad de adaptación a eventos de aumento de temperatura vinculado con el crecimiento de la planta. Zhang et al. (2014) encontraron que con un incremento de temperatura entre 0.4 a $1.7{ }^{\circ} \mathrm{C}$, aumentaba la abundancia de todos los grupos microbianos, excepto de los protozoos. Los microorganismos del suelo tienden a presentar un comportamiento distinto a eventos de aumento de temperatura; mientras que algunos microorganismos pueden aumentar su crecimiento otros disminuyen. Lo anterior, relacionado con factores como la capacidad de termo-adaptación, cambios en la productividad de la planta, composición química, cantidad y calidad de la materia orgánica, adaptación evolutiva, pérdida del carbono lábil del suelo, entre otros (Wang et al., 2017; Creamer et al., 2015; Bradford, Watts, \& Davies, 2010; RomeroOlivares, Allison, \& Treseder, 2017).

En esta investigación un aumento en la temperatura de $5.1^{\circ} \mathrm{C}$, afectó negativamente la reproducción de las plantas, la biomasa radical y las BFN, pero favoreció una mayor acumulación de materia seca en la parte aérea y un incremento en la fotosíntesis $(A)$.

Declaración de ética: los autores declaran que todos están de acuerdo con esta publicación y que han hecho aportes que justifican su autoría; que no hay conflicto de interés de cualquier tipo; y que han cumplido con todos los requisitos y procedimientos éticos y legales pertinentes. El documento firmado se encuentra en los archivos de la revista.

\section{AGRADECIMIENTOS}

La presente investigación fue financiada por el proyecto de desarrollo científico para atender problemas nacionales CONACYT 213059 "Impacto del cambio climático y la actividad agrícola en la emisión de gases de efecto de invernadero y en los recursos microbianos de la Sierra Nevada, México".

\section{RESUMEN}

Para los próximos 100 años se pronostica un incremento en la temperatura del planeta de casi $4{ }^{\circ} \mathrm{C}$, lo cual pondrá en riesgo las especies que no logren adaptarse. En esta investigación se determinaron las respuestas morfofisiológicas de $F$. mexicana y los cambios en la población de bacterias fijadoras de nitrógeno atmosférico (BFN) asociadas a sus raíces, debido a dos condiciones ambientales con diferencias medias de temperatura de $5.1^{\circ} \mathrm{C}$ : invernadero (temperatura alta, TA) y campo abierto (temperatura baja, TB); y con cuatro tratamientos: sin inocular (T), inoculadas con la cepa ocho (CP8), cepa cuatro (CP4) y con ambas cepas $(\mathrm{CP} 8+4)$. Las BFN fueron aisladas de la rizosfera de F. mexicana y transformadas genéticamente con reporteros, para cuantificar la población al final del experimento. Se midió el peso seco de la parte aérea y la raíz, la tasa de asimilación de $\mathrm{CO}_{2}(A)$, el inicio de la floración, el número de flores y frutos; y la persistencia de las bacterias fijadoras de $\mathrm{N}$ atmosférico (BFN). Además, se evaluó la concentración de $\mathrm{NO}_{3}, \mathrm{NH}_{4}$, $\mathrm{P}$ y materia orgánica (MO) en el sustrato, al inicio y final del experimento. Las plantas sometidas a alta temperatura presentaron mayor peso seco de la parte aérea y fotosíntesis; con una disminución en el peso seco de la raíz, y en el número de flores y frutos. La MO en el sustrato disminuyó, mientras que la disponibilidad de $\mathrm{NO}_{3}$, $\mathrm{NH}_{4}$ y P aumentó. El incremento de temperatura y la mayor presencia de $\mathrm{N}$ en el substrato provocaron reducción en la población de BFN. Estos resultados sugieren que temperaturas altas estimulan el crecimiento de F. mexicana y tienen un impacto negativo sobre su reproducción y en las BFN asociadas a sus raíces.

Palabras clave: calentamiento global; fotosíntesis; crecimiento; materia orgánica; bacterias fijadoras de nitrógeno. 


\section{REFERENCIAS}

Asseng, S., Foster, I., \& Turner, N. (2011). The impact of temperature variability on wheat yields. Global Change Biology, 17, 997-1012. DOI:10.1111/j.1365-2486.2010.02262.x

Åström, H., Metsovuori, E., Saarinen, T., Lundell, R., \& Hänninen, H. (2015). Morphological characteristics and photosynthetic capacity of Fragaria vesca L. winter and summer leaves. Flora-Morphology, Distribution, Functional Ecology of Plants, 215, 33-39. DOI: 10.1016/j.flora.2015.07.001

Baker, A. S. (1969). Colorimetric determination of nitrate in soil and plant extracts with brucine. Journal of Agriculture and Food Chemistry, 17, 802-806. DOI: 10.1021/jf60153a004

Barrie, F. R. (2015). Rosaceae. En G. Davidse, M. SousaSánchez, S. Knapp, \& F. Chiang-Cabrera (Eds.), Flora Mesoamericana (Volumen 2, Parte 3, pp. 1-110). México, USA y UK: Universidad Nacional Autónoma de México, Instituto de Biología, Missouri Botanical Garden y Natural History Museum. Recuperado de http://worldfloraonline.org/taxon/ wfo-0000993770; jsessionid=813FBD58AC1DB708 12BA2C8B7E7750F5

Benlloch-González, M., Quintero, J. M., Suárez, M. P., Sánchez-Lucas, R., Fernández-Escobar, R., \& Benlloch, M. (2016). Effect of moderate high temperature on the vegetative growth and potassium allocation in olive plants. Journal of Plant Physiology, 207, 22-29. DOI: $10.1016 /$ j.jplph.2016.10.001

Blümel, M., Dally, N., \& Jung, C. (2015). Flowering time regulation in crops-what did we learn from Arabidopsis? Current Opinion in Biotechnology, 32, 121-129. DOI: $10.1016 /$ j.copbio.2014.11.023

Bonilla-Valencia, L., Martínez-Orea, Y., Castillo-Argüero, S., Barajas-Guzmán, G., Romero-Romero, M. A., \& Díaz-López, E. T. (2017). Reproductive phenology of understory species in an Abies religiosa (Pinaceae) forest in the Magdalena River Basin, Mexico City. Journal of the Torrey Botanical Society, 144(3), 313327. DOI: 10.3159/TORREY-D-16-00024.1

Bradford, M. A., Watts, B. W., \& Davies, C. (2010). Thermal adaptation of heterotrophic soil respiration in laboratory microcosms. Global Change Biology, 16, 1576-1588. DOI: 10.1111/j.1365-2486.2009.02040.x

Bray, R. H., \& Kurtz, L. T. (1945). Determination of total, organic and available forms of phosphorus in soil. Soil Science, 59, 39-45.

Chabot, B. F. (1978). Environmental influences on photosynthesis and growth in Fragaria vesca. New Phytologist, 80, 87-98. DOI: 10.1111/j.14698137.1978.tb02267.x

Compant, S., van der Heijden, M. G. A., \& Sessitsch, A. (2010). Climate change effects on beneficial plant-microorganism interactions. Minireview. FEMS, Microbiology Ecology, 73, 197-214. DOI: 10.1111/j.1574-6941.2010.00900.x

Creamer, C. A., de Menezes, A. B., Krull, E. S., Sanderman, J., Newton-Walters, R., \& Farrell, M. (2015). Microbial community structure mediates response of soil $\mathrm{C}$ decomposition to litter addition and warming. Soil Biology \& Biochemistry, 80, 175-188. DOI: 10.1016/j.soilbio.2014.10.008

Egamberdiyeva, D., \& Höflich, G. (2003). Influence of growth-promoting bacteria on the growth of wheat in different soils and temperatures. Soil Biology \& Biochemistry, 35, 973-978. DOI: 10.1016/ S0038-0717(03)00158-5

Greer, D. H., \& Weedon, M. M. (2012). Modelling photosynthetic responses to temperature of grapevine (Vitis vinifera cv. Semillon) leaves on vines grown in a hot climate. Plant, Cell and Environment, 35, 1050-1064. DOI: 10.1111/j.1365-3040.2011.02471.x

Gregory, P. J., \& Kirkegaard, J. A. (2017). Growth and Function of Root Systems. In B. Thomas, B. G. Murray, \& D. J. Murphy (Eds.), Encyclopedia of Applied Plant Sciences ( $2^{\text {nd }}$ ed, pp. 230237). Netherlands: Elsevier Ltd. DOI: $10.1016 / \mathrm{j}$. envexpbot.2017.03.001

Hartsan, J., Pavlovic, L., Chiu, G., Sultmanis, S., \& Sage, T. L. (2013). High temperature stress and its effect on pollen development and morphological components of harvest index in the $\mathrm{C}_{3}$ model grass Brachypodium distachyon. Journal of Experimental Botany, 64(10), 2971-2983. DOI: 10.1093/jxb/ert142

Huang, H., von Lampe, M., \& van Tongeren, F. (2011). Climate change and trade in agriculture. Food Policy, 36, S9-S13. DOI: 10.1016/j.foodpol.2010.10.008

Ledesma, N. A., \& Kawabata, S. (2016). Responses of two strawberry cultivars to severe high temperature stress at different flower development stages. Scientia Horticulturae, 211, 319-327. DOI: 10.1016/j. scienta.2016.09.007

Li, Q., Cheng, X., Luo, Y., Xu, Z., \& Xu, X. (2017). Consistent temperature sensitivity of labile soil organic carbon mineralization along an elevation gradient in the Wuyi Mountains, China. Applied Soil Ecology, 117-118, 32-37. DOI: 10.1016/j.apsoil.2017.04.018

Li, X., Takahashi, T., Suzuki, N., \& Kaiser, H. M. (2014). Impact of Climate Change on Maize Production in Northeast and Southwest China and Risk Mitigation Strategies. Asia-Pacific Chemical, Biological \& Environmental Engineering, APCBEE Procedia, 8, 11-20. DOI: 10.1016/j.apcbee.2014.01.073

Liu, Y., Mu, J., Niklas, K. J., Li, G., \& Sun, S. (2012). Global warming reduces plant reproductive output for temperate multi-inflorescence species on the Tibetan plateau. New Phytologist, 195, 427-436. DOI: $10.1111 / \mathrm{j} .1469-8137.2012 .04178 . x$ 
Lizana, X. C., Avila, A., Tolaba, A., \& Martínez, J. P. (2017). Field responses of potato to increased temperature during tuber bulking: Projection for climate change scenarios, at high-yield environments of Southern Chile. Agricultural and Forest Meteorology, 239, 192-201. DOI: 10.1016/j.agrformet.2017.03.012

Lodish, H., Berk, A., Zipursky, S. L., Matsudaira, P., Baltimore, D., \& Darnell. J. (2000). DNA Cloning with Plasmid Vectors. In H. Lodish, A. Berk, S. L. Zipursky, P. Matsudaira, D. Baltimore, \& J. Darnell (Eds.), Molecular Cell Biology (4 ${ }^{\text {th }}$ ed.). Nueva York, E.U.A: W. H. Freeman and Company Eds. Recuperado de https://www.ncbi.nlm.nih.gov/books/NBK21498

Merlo, C., Reyna, L., Abril, A., Amé, M. V., \& Genti-Raimondi, S. (2014). Environmental factors associated with heterotrophic nitrogen-fixing bacteria in water, sediment, and riparian soil of Suquía River. Limnologica - Ecology and Management of Inland Waters, 48, 71-79. DOI: 10.1016/j.limno.2014.06.004

Mittermeier, R., \& Goettsch, C. (1992). La importancia de la diversidad biológica de México. En J. Sarukhán, \& R. Dirzo (Eds.), México ante los retos de la biodiversidad (pp. 43-55). México: Comisión Nacional para el Conocimiento y Uso de la Biodiversidad, CONABIO.

Nakano, Y., Higuchia, Y., Yoshidab, Y., \& Hisamatsua, T. (2015). Environmental responses of the FT/TFL1 gene family and their involvement in flower induction in Fragaria $\times$ ananassa. Journal of Plant Physiology, 177, 60-66. DOI: 10.1016/j.jplph.2015.01.007

Ortiz-Solorio, C., \& Cuanalo de la Cerda, H. E. (1977). Levantamiento Fisiográfico del Área de Influencia de Chapingo: Para la Cartografía de Tierras Erosionadas. Texcoco, México: Colegio de Postgraduados, Escuela Nacional de Agricultura, Chapingo.

Paul, E. (2007). Soil Microbiology, Ecology and Biochemistry. San Diego, Estados Unidos: Academic Press, Inc.

Pawar, K. V., \& Rothkar, R. V. (2015). Forest Conservation \& Environmental Awareness. Procedia Earth and Planetary Science, 1, 212 -215. DOI: 10.1016/j. proeps.2015.06.027

Porch, T. G., \& Jahn, M. (2001). Effects of high-temperature stress on microsporogenesis in heat-sensitive and heat-tolerant genotypes of Phaseolus vulgaris. Plant, Cell and Environment, 24, 723-731. DOI: 10.1046/j.1365-3040.2001.00716

Prasad, P. V., \& Djanaguiraman, M. (2014). Response of floret fertility and individual grain weight of wheat to high temperature stress: sensitive stages and thresholds for temperature and duration. Functional Plant Biology, 41, 1261-1269. DOI: 10.1071/FP14061

Prasad, P., Thomas, J. M. G., \& Narayanan, S. (2017). Global Warming Effects. In B. Thomas, B. G. Murray, \& D. J. Murphy (Eds.), Encyclopedia of
Applied Plant Sciences (2 ${ }^{\text {nd }}$ ed., Vol. 3, pp. 289299). Netherlands: Elsevier Ltd. DOI: 10.1016/ B978-0-12-394807-6.00013-7

R Development Core Team. (2018). R: A language and environment for statistical computing. Vienna, Austria: R Foundation for Statistical Computing. Recuperado de https://www.r-project.org

Rennie, R. J. (1981). A single medium for the isolation of acetylene-reducing (dinitrogen-fixing) bacteria from soils. Canadian Journal of Microbiology, 27, 8-14. DOI: $10.1139 / \mathrm{m} 81-002$

Robert, F., Risser, G., \& Pétel, G. (1999) Photoperiod and temperature effect on growth of strawberry plant (Fragaria $\times$ ananassa Duch.): development of a morphological test to assess the dormancy induction. Scientia Horticulturae, 82, 217-226.

Romero-Olivares, A. L., Allison, S. D., \& Treseder, K. K. (2017). Review Paper: Soil microbes and their response to experimental warming over time: A metaanalysis of field studies. Soil Biology \& Biochemistry, 107, 32-40. DOI: 10.1016/S1002-0160(14)60029-1

Rothstein, D. E., \& Zack, D. R. (2001). Photosynthetic adaptation and acclimation to exploit seasonal periods of direct irradiance in three temperate, deciduousforest herbs. Functional Ecology, 15, 722-731. DOI: 10.1046/j.0269-8463.2001.00584.x

Rzedowski, J., \& Calderón, G. (2005). Rosacea. En J. Rzedowski, \& G. Calderón de Rzedowski (Eds.), Flora del Bajio y de Regiones Adyacentes (Fascículo 135, pp. 60-64). Pátzcuaro, Michoacán, México: Instituto de Ecología, A.C., Centro Regional del Bajío.

Saarinen, T., Rasmus, S., Lundell, R., Kauppinen, O. K., \& Hänninen, H. (2016). Photosynthetic and phenological responses of dwarf shrubs to the depth and properties of snow. Oikos, 125(3), 364-373. DOI: 10.1111/oik.02233

Sage, T., Bagha, S., Lundsgaard-Nielsen, V., Branch, H. A., Sultmanis, S., \& Sage, R. F. (2015). The effect of high temperature stress on male and female reproduction in plants. Field Crops Research, 182, 30-42. DOI: 10.1016/j.fcr.2015.06.011

Sambrook, J., Maniatis, T., \& Fritsch, E. T. (1989). Molecular Cloning, a Laboratory Manual (2 $2^{\text {nd }}$ ed.). New York, EE.UU: Cold Spring Harbor Laboratory Press.

Sánchez-González, A., López-Mata, L., \& Vibrans, H. (2006). Composición y patrones de distribución geográfica de la flora del bosque de oyamel del cerro Tláloc, México. Boletín de la Sociedad Botánica de México, 79, 67-78.

Sheehy, J. E., Mitchell, P. L., \& Ferrer, A. B. (2006). Decline in rice grain yields with temperature: Models and correlations can give different estimates. Field Crops Research, 98, 151-156. DOI: 10.1016/j. fcr.2006.01.001 
Shen, R. C., Xu, M., Chi, Y. G., Yu, S., \& Wan, S. G. (2014). Soil microbial responses to experimental warming and nitrogen addition in a temperate steppe of Northern China. Pedosphere, 24(4), 427-436. DOI: 10.1016/S1002-0160(14)60029-1

Sierra, J., Brisson, N., Ripoche, D., \& Déqué, M. (2010). Modelling the impact of thermal adaptation of soil microorganisms and crop system on the dynamics of organic matter in a tropical soil under a climate change scenario. Ecological Modelling, 221, 2850-2858. DOI: 10.1016/j.ecolmodel.2010.08.031

Sofi, J. A., Lone, A. H., Ganie, M. A., Dar, N. A., Bhat, A. A., Mukhtar, M., ... Ramzan, S. (2016). Soil Microbiological Activity and Carbon Dynamics in the Current Climate Change Scenarios: A Review. Pedosphere, 26(5), 577-591. DOI: 10.1016/ S1002-0160(15)60068.6

Sønsteby, A., Opstada, N., \& Heideb, O. M. (2013). Environmental manipulation for establishing high yield potential of strawberry forcing plants. Scientia Horticulturae, 157, 65-73. DOI 10.1016/j. scienta.2013.04.014

Sønsteby, A., Solhaug, K. A., \& Heide, O. M. (2016). Functional growth analysis of 'Sonata' strawberry plants grown under controlled temperature and daylength conditions. Scientia Horticulturae, 211, 26-33. DOI: 10.1016/j.scienta.2016.08.003

Statistical Analysis System (SAS). (2002). User's Guide (Version 9). Cary, North Carolina, USA: SAS Institute Inc.

Struik, P. C. (2007). Responses of the Potato Plant to Temperature. In D. Vreugdenhil (Ed.), Potato Biology and Biotechnology: Advances and Perspectives (pp. $367-$ 393). Nueva York, E.U.A.: Elsevier B.V.

Tkemaladze, G. S., \& Makhashvili, K. A. (2016). Climate changes and photosynthesis. Annals of Agrarian Science, 14, 119-126. DOI: 10.1016/j. aasci.2016.05.012

Torres, J. M. (2004). Estudio de tendencias y perspectivas del sector forestal en América Latina al año 2020 (Informe Nacional México). Roma, Italia: Organización de las Naciones Unidas para la Agricultura y la Alimentación, FAO.

Tripathi, V., Abhilash, P. C., Singh, H. B., Singh, N., \& Patra, D. D. (2015). Effect of temperature variation on lindane dissipation and microbial activity in soil. Ecological Engineering, 79, 54-59. DOI: 10.1016/j. ecoleng.2015.03.010

Urbanová, M., Snajdr, J., \& Baldrian, P. (2015). Composition of fungal and bacterial communities in forest litter and soil is largely determined by dominant trees. Soil Biology \& Biochemistry, 84, 53-64. DOI: 10.1016/j.soilbio.2015.02.011

Vanha-Majamaa, I., Shorohova, E., Kushnevskaya, H., \& Jalonen, J. (2017). Resilience of understory vegetation after variable retention felling in boreal Norway spruce forests - A ten-year perspective. Forest Ecology and Management, 393, 12-28. DOI: 10.1016/j. foreco.2017.02.040

Villaseñor, J. L., \& Ortiz, E. (2014). Biodiversidad de las plantas con flores (División Magnoliophyta) en México. Revista Mexicana de Biodiversidad, 85, S134-S142. DOI: 10.7550/rmb.31987

Villaseñor, J. L. (2016). Checklist of the native vascular plants of Mexico. Revista Mexicana de Biodiversidad, 87, 559-902. DOI: 10.1016/j.rmb.2016.06.017

Walkley, A., \& Black, I. A. (1934). An examination of Degtjareff method for determining soil organic matter and a proposed modification of the chromic acid titration method. Soil Science, 37, 29-38.

Wang, C., Xinquan, Z., Hongbiao, Z., Lei, H., Luji, A., Wang, G., \& Lerdau, M. (2017). The effect of simulated warming on root dynamics and soil microbial community in an alpine meadow of Qinghai-Tibet Plateau. Applied Soil Ecology, 116, 30-41. DOI: 10.1016/j.apsoil.2017.03.005

Wang, G. B., Zhou, Y., Xu, X., Ruan, H. H., \& Wang, J. S. 2013. Temperature sensitivity of soil organic carbon mineralization along an elevation gradient in the Wuyi Mountains, China. PLOS One 8(1), 1-7. DOI: 10.1371/journal.pone.0053914

Wetterstedt, J. A. M., Persson, T., \& Agren, G. I. (2010). Temperature sensitivity and substrate quality in soil organic matter decomposition: results of an incubation study with three substrates. Global Change Biology, 16(6), 1806-1819. DOI: 10.1111/j.1365-2486.2009.02112.x

Yanagi, T., Takanori, Y., \& Ncbuyuki, O. (2006). Photoperiodic reaction of sexual and asexual reproduction in Fragaria chiloensis L. CHI-24-1 plants grown at various temperatures. Scientia Horticulturae, 110(2), 187-191. DOI: 10.1016/j.scienta.2006.07.004

Young, L. W., Wilen, R. W., \& Bonham-Smith, P. C. (2004). High temperature stress of Brassica napus during flowering reduces micro- and megagametophyte fertility, induces fruit abortion, and disrupts seed production. Journal of Experimental Botany, 55(396), 485-495. DOI: 10.1093/jxb/erh038

Zhang, Y., Cai, Y., Beach, R. H., \& McCarl, B. A. 2014. Modeling Climate Change Impacts on the US Agricultural Exports. Journal of Integrative Agriculture, 13(4), 666-676. DOI: 10.1016/ S2095-3119(13)60699-1 